\title{
Raloxifene Treatment Enhances Brain Activation during Recognition of Familiar Items: a Pharmacological fMRI Study in Healthy Elderly Males
}

\author{
Rutger Goekoop*,', Frederik Barkhof ${ }^{2}$, Erik JJ Duschek ${ }^{3}$, Coen Netelenbos ${ }^{3}$, Dirk L Knol ${ }^{4}$, Philip Scheltens' \\ and Serge ARB Rombouts ${ }^{1,5}$ \\ 'Department of Neurology, VU University Medical Center, Amsterdam, The Netherlands; ${ }^{2}$ Department of Radiology, VU University Medical \\ Center, Amsterdam, The Netherlands; ${ }^{3}$ Department of Endocrinology, VU University Medical Center, Amsterdam, The Netherlands; ${ }^{4}$ Department \\ of Statistics, VU University Medical Center, Amsterdam, The Netherlands; ${ }^{5}$ Department of Physics \& Medical Technology, VU University Medical \\ Center, Amsterdam, The Netherlands
}

Raloxifene is a selective estrogen receptor modulator that may delay the onset of mild cognitive impairment in elderly women. Effects of raloxifene treatment on mental performance in males remain to be investigated. In a previous functional magnetic resonance imaging (fMRI) study, we showed that raloxifene treatment enhanced brain activation in elderly males during encoding of new information (faces) into memory. The current study used $\mathrm{PMRI}$ in the same group of subjects to screen for effects of raloxifene treatment on brain function during face recognition. Healthy elderly males $(n=28$; mean age 63.6 years, SD 2.4) were scanned at baseline and after 3 months of treatment with either raloxifene $120 \mathrm{mg}(n=14)$ or placebo $(n=14)$ in a randomized, double-blind, placebo-controlled study design. Functional data were analyzed in an event-related fashion with respect to correct hits and correct rejections using FSL software. Performance data were analyzed with respect to recognition accuracy, latency, and response bias. Functional effects of treatment were found on brain activation related to correct hits only. When compared to placebo treatment, raloxifene treatment enhanced brain activation in the left posterior parahippocampal area $(Z=3.9)$ and right inferior prefrontal cortex $(Z=3.5)$. Recognition accuracy scores remained stable in the raloxifene group, whereas the placebo group showed a small but significant decrease in accuracy scores $(p=0.02)$. No significant effects were found on response bias or latency. In conclusion, raloxifene treatment affects brain function during memory performance in a way that may reflect increased arousal during initial encoding, with downstream effects on brain function during retrieval of information. Behaviorally, such neurofunctional effects may actively block decreased memory performance as a result of contextdependency. The validity of these predictions can be tested in large-scale clinical trials.

Neuropsychopharmacology (2006) 3 I, I508-1518. doi:I0.1038/sj.npp. I300956; published online 16 November 2005

Keywords: fMRI; SERM; raloxifene; male; memory; recognition

\section{INTRODUCTION}

Raloxifene is a selective estrogen receptor modulator (SERM) that is used to treat osteoporosis in postmenopausal women (Heringa, 2003). Raloxifene has positive effects on cardiovascular risk factors, and is not associated with an increased risk of stroke and cardiovascular disease that is observed for regular estrogen treatment (Barrett-Connor et al, 2002). For such reasons, raloxifene treatment is

\footnotetext{
*Correspondence: Dr R Goekoop, Department of Neurology, VU University Medical Center, De Boelelaan II 17 1081 HV, Amsterdam, The Netherlands, Tel: +3I 2044403 I6, Fax: + 31204440317 , E-mails: R.Goekoop@VUMC.nl; rgoekoop@dds.nl

Received 5 July 2005; revised 7 September 2005; accepted 27 September 2005

Online publication: 6 October 2005 at http://www.acnp.org/citations/ Npp I00605050434/default.pdf
}

considered an attractive alternative for prevention and treatment of osteoporosis and heart disease in male subjects as well (Doran et al, 2001; Blum et al, 2000). Recent results from a randomized controlled clinical trial show that raloxifene treatment at $120 \mathrm{mg}$ daily for 3 years may successfully delay the onset of mild cognitive impairment (MCI) and possibly Alzheimer's Disease (AD) in elderly women (Yaffe et al, 2005). Thus, the need for an assessment of the effects of raloxifene treatment on male brain function and mental performance is growing.

Estrogens and SERMs such as raloxifene may differentially affect mental performance, including memory performance, in both sexes (Cahill, 2003). It is therefore unclear whether the preventive effects of raloxifene treatment in women can be extrapolated to males. To our knowledge, no studies have been published that examined effects of raloxifene treatment on memory performance in male 
subjects. Such studies usually involve large-scale clinical trials in which subjects or patients are examined for several years in order to detect significant effects of pharmacological treatment on neuropsychological measures of behavior. While anticipating the results of such studies, the current study examined the effects of 3 months of raloxifene treatment on brain function during memory task performance using functional magnetic resonance imaging (fMRI). Task-related fMRI yields both functional and behavioral measures of treatment effects, which may serve as preliminary findings prior to large-scale investigations of similar effects in controlled clinical trials. fMRI may be more sensitive and less ambiguous to the effects of sex steroids on mental performance than conventional neuropsychological assessment scales (Maki and Resnick, 2001). Additionally, fMRI may provide an insight into the treatment mechanism of raloxifene. Treatment effects on brain function can be mapped to individual brain structures at high spatial resolution (Honey and Bullmore, 2004). The behavioral significance of such effects may be examined either by direct correlation of behavioral measures with such effects (Goekoop et al, 2005), or by examining brain function associated with distinct response types during memory performance (eg correct hits, correct rejections) (Buckner et al, 1998). This allows a detailed analysis of the effects of SERMs on memory function, which may eventually be relevant to the design and development of new SERMs that target specific aspects of memory performance, such as encoding and retrieval processes (Bernardi et al, 2003).

In a previous fMRI study, we examined effects of raloxifene treatment $v s$ placebo on brain function in healthy elderly males during encoding of new information (human faces) into memory (Goekoop et al, 2005). Raloxifene globally enhanced brain activation during face encoding, with the exception of subcortical, medial temporal, and visual structures. This was interpreted as a global effect of raloxifene treatment on cortical arousal, rather than a specific influence on each of the cognitive domains represented by this pattern (Goekoop et al, 2005). Such effects are in line with findings from a large number of studies, suggesting that estrogens have a context-dependent effect on cortical arousal (Morgan et al, 2004; Cahill and Alkire, 2003). Based on the locations of treatment effects, we predicted small effects of raloxifene treatment on working memory performance, executive functions, and verbal skills (Goekoop et al, 2005).

Since no treatment effects were found in subcortical or medial temporal areas during encoding (despite activation of these structures during task performance), we considered an effect of raloxifene treatment on episodic memory to be less likely. However, the effects of raloxifene treatment on episodic memory could not be fully assessed, since only the encoding phase of memory performance was examined. Since a growing number of imaging studies has shown that pharmacological substances affect brain function in process-specific manner (Honey and Bullmore, 2004; Thiel, 2003), raloxifene treatment may have unique effects on brain function during encoding, consolidation, or retrieval stages of memory performance. We therefore used fMRI to examine the effects of 3 months of raloxifene treatment on brain function during face recognition. Given the reported efficacy of raloxifene treatment in preventing the onset of memory impairment in elderly women, we hypothesized that raloxifene treatment would eventually affect brain function during retrieval of episodic information.

\section{METHODS}

\section{Study Design}

Subjects were screened for participation in a randomized, double-blind, placebo-controlled repeated measures design. In each subject, fMRI was performed at baseline (BL; no medication; session 1) and after 3 months of a once daily oral intake of raloxifene $120 \mathrm{mg}$ or placebo (TR; session 2). This dose was chosen because a previous study showed no significant effects of raloxifene $60 \mathrm{mg}$ on markers of bone turnover in males (Doran et al, 2001). BL and TR sessions were performed on the same time of day in each subject (margin $30 \mathrm{~min}$ ). If data acquisition failed, the subject's consent was asked for an additional scanning visit, up until which time the relevant medication regime (raloxifene or placebo) was continued, to obtain a maximum number of complete data sets. Study period extension was not to exceed 10 days.

\section{Subject Recruitment}

The medical ethical review board of the VU University Medical Center of Amsterdam approved the study. A total of 30 healthy, right-handed elderly males, aged 60-70 years (mean 63.6 years, SD 2.4; range 60-69 years) were recruited by advertisement in local newspapers. This was the same study population that was examined previously for effects of raloxifene treatment on brain function during face encoding. All subjects provided informed consent during a screening visit in which the procedure was explained and contraindications were checked. The mental status of subjects was assessed by means of a structured clinical interview. This interview involved questions regarding the subjects' general health, mental health, social status, history, and intoxications. Exclusion criteria involved (a history of) psychiatric disease (ie any diagnosable disorders according to DSM-4-TR criteria (American Psychiatric Association, 2000), any neurological illness (memory complaints in particular), and the use of any medication or other substances that are known to influence cerebral functioning (including $>0.5$ pack of cigarettes, $>4$ glasses of alcohol). Elaborate physical examination and laboratory tests were performed to back the clinical interview. Exclusion criteria to MRI involved the presence of metallic implants in highrisk areas and a history of claustrophobia. Formal education was determined using a dutch system (low, middle, high). On the day of the second scanning session (after 3 months), a similar structured clinical interview was performed with a particular focus on side effects of treatment. Compliance was assessed by pill counts and the subjects' comments.

\section{fMRI}

Imaging was carried out on a $1.5 \mathrm{~T}$ Sonata MR scanner (Siemens, Erlangen, Germany), using a standard circularly polarized head coil with foam padding to restrict head 
motion. For fMRI, an echo planar imaging sequence was used (echo time $60 \mathrm{~ms}$, flip angle $90^{\circ}$, matrix $64 \times 64$, field of view $192 \times 192 \mathrm{~mm}$ ) to obtain 21 transverse slices (thickness $5 \mathrm{~mm}$, interslice gap $1 \mathrm{~mm}$ ). Task stimuli were projected on a screen located at the head end of the scanner table via an LCD projector located outside the scanner room. Subjects viewed the screen through a mirror located on the head coil. In each hand, subjects held an fMRI compatible responsebox through which they were able to react to task stimuli by pressing a single button using one of their index fingers. A T1-weighted structural MRI scan was obtained of each subject (MPRAGE; inversion time: $300 \mathrm{~ms}$, TR $=15 \mathrm{~ms}$; $\mathrm{TE}=7 \mathrm{~ms}$; flip angle $=8^{\circ} ; 160$ coronal slices, $1 \times 1 \times 1.5 \mathrm{~mm}$ voxels). Total scanning time including structural imaging on average was $21 \mathrm{~min}$ for each visit.

\section{Face Recognition Task}

Immediately after face encoding, which involved the presentation of four blocks of unfamiliar faces (24 in total), alternating with blocks of fixation (Goekoop et al, 2005), a recognition task was administered. Total scanning time was $6 \mathrm{~min} 12 \mathrm{~s}$ for the encoding task and $3 \mathrm{~min} 40 \mathrm{~s}$ for the recognition task. Hence, minimum delay between items presented during encoding and recognition tasks was $\sim 1 \mathrm{~min}$, and maximum delay $\sim 10 \mathrm{~min}$. During the first $10.5 \mathrm{~s}$ of each memory-task, subjects saw a circle indicating time left before the onset of the first condition. During face recognition, 24 faces, of which 12 had been shown during encoding and 12 were new, were presented sequentially in random order on a black background. Presentation time was $5 \mathrm{~s}$ for each face, followed by a white fixation-cross presented for $3 \mathrm{~s}$ on a black background. We chose to contrast faces with a 'low level' fixation cross (X) in order to maximize contrast between processing demands for both conditions, which would result in a large number of brain areas being activated for faces $>$ fixation contrasts, including visual and sensorimotor areas. This would increase chances of finding significant effects of treatment. Subjects were instructed verbally to indicate whether the presented faces were familiar or unfamiliar by pressing one of two buttons (written instructions 'Left: familiar' and 'Right: unfamiliar' also appeared alongside the pictures). Mean reaction times and the number of hits and misses were recorded. To avoid investigator's bias, task performance data were viewed only after both scanning visits had been completed.

Two comparable versions of each paradigm were constructed and randomized across subjects and scanning sessions (baseline, treatment) to reach equal numbers of subjects receiving test versions 1 and 2 in both treatment groups. A third 'spare' version was available in case data acquisition might fail during one of the scanning sessions. Each paradigm contained unique items (faces) to avoid any overlap or interference with previously observed items at the time of the second scanning session. Paradigms were practised extensively to minimize effects of skill learning in the course of the scanning sessions. A practicing task was used that was similar to tasks used for neuroimaging, but contained less items (practicing items did not overlap with those of tasks used for scanning purposes). A day before the onset of the first scanning session, subjects visited the hospital and practiced both encoding and retrieval tasks. On the day of the first scanning session, minutes before the onset of the first measurements, the tasks were again practiced with subjects in the scanner. On the day of the the second scanning session, subjects practiced the procedure within the scanner only. Total time for one scanning visit including instructions of memory tasks was approximately $1 \mathrm{~h}$.

\section{Statistical Analysis of Task Performance Data}

Post hoc sorting of response types yielded a number of true positive (TP, correct hits), true negative ( $\mathrm{TN}$, correct rejections), false positive (FP, false hits), false negative (FN, false rejections), and forgotten items (Forgot). Based on these responses, an overall performance accuracy score ('Pr') was calculated by using the following formula: $\mathrm{Pr}=\mathrm{FP}-\mathrm{TP}$ (Corwin, 1994). This measure was divided by the total number of new items (12) to correct for $50 \%$ chance levels, yielding scores between -1 and 1 . A 'falsealarm rate' ('FAR') was calculated using the following formula: $\mathrm{FAR}=(\mathrm{FP}+0.5) / 13$. A measure of response bias ' $\mathrm{Br}$ ' was then calculated using the following formula: $\mathrm{Br}=\mathrm{FAR} /(1-\mathrm{Pr})$. This measure indicates the chance that subjects will guess that tested items are targets when they are in an uncertain state. High values of $\mathrm{Br}$ indicate a tendency to produce high numbers of FP responses, whereas low values of $\mathrm{Br}$ indicate high uncertainty (Corwin, 1994).

Three independent variables were examined for effects of raloxifene vs placebo treatment: that is, accuracy (Pr), latency (RTav), and response bias $(\mathrm{Br})$. For each variable, a single mixed effects (ME) linear model was specified using SPSS 12.0, with 'group' (two levels: RAL, PLAC), 'scanorder' (two levels: session 1, session 2), and 'test version' (two levels: 1 and 2) as fixed factors, 'scanorder' being the repeated factor, and 'subjectnr' defining linked measurements from the same subject. This model allowed calculation of significant effects of raloxifene $v s$ placebo treatment (interaction group ${ }^{*}$ scanorder), as well as effects of raloxifene or placebo treatment separately (custom contrasts examining 'placebo: session 2-session 1', and 'raloxifene: session 2 - session 1') and differences in performance between raloxifene and placebo groups at baseline and after treatment (custom contrasts examining 'session1: RAL-PLAC', and 'session 2: RAL-PLAC'). Effects were examined using a threshold for significance defined by $p<0.05$, Bonferroni corrected for multiple comparisons. The advantage of this model is that it is more accurate than conventional models, allows calculation of all effects of interest within the confines of a single statistical model, and is closely related to the model used for analysis of the functional data. Since our previous analysis of the same performance data used the conventional but less accurate (multimodel) approach, the significance of the observed effects as reported in the current study may vary to some degree with those reported earlier (Goekoop et al, 2005). However, the basic trends remain the same (see Results section).

\section{Analysis of Functional Neuroimaging Data}

Functional data sets were analyzed using FSL 3.2 (Smith et al, 2004). The first five volumes of each data set were 
discarded to account for T1-saturation effects. At first level (individuals), the following preprocessing was applied: nonbrain removal, slice-timing correction using Fourier-space time-series phase-shifting, motion correction and spatial smoothing using a Gaussian kernel of FWHM $8 \mathrm{~mm}$, meanbased intensity normalization of all volumes by the same factor, and high and low pass temporal filtering (Jenkinson et al, 2002; Smith, 2002). Functional neuroimages of each subject were coregistered to corresponding structural images in native space, and structural images were registered to structural Talairach standard images (Talairach and Tournoux, 1988), defined by the Montreal Neurological Institute standard brain supplied with FSL. The same transformation matrices used for structural-to-standard transformations were then used for functional-to-standard space transformations of coregistered functional images. All registrations were carried out using an intermodal registration tool based on the correlation ratio (Jenkinson and Smith, 2001). After preprocessing, the following statistics was applied on a voxel-wise basis on each time series, using local autocorrelation correction (Woolrich et al, 2001): signal change during face recognition was modeled in an eventrelated fashion, using separate regressors for TP, TN, FP, FN, and forgotten response types (see above). Signal variance during fixation ( $\mathrm{X}$ condition) was not modeled, to prevent overspecification of the model. Type and onset time of the events were determined by post hoc sorting, based on the responses given by the individual subjects. Thus, all individuals obtained a unique model of signal response containing a regressor for each response type, which was convolved with a $\gamma$ function to model the hemodynamic response. Model fitting generated whole brain images in native space of parameter estimates and corresponding variance, representing average signal change during a particular condition (eg TP, TN, FP, FN, forgot) vs fixation (X, an implicit baseline condition). Owing to a limited number of false responses, effects of raloxifene treatment $v s$ placebo were examined on 'TP', 'TN', 'TP $>\mathrm{TN}$ ', and 'TN $>$ TP' contrasts only, that is, false responses were ignored in further analyses.

Since our face recognition task contained both novel and familiar items, both encoding and retrieval processes occurred during task performance (Buckner et al, 2001). Such processes can be studied separately by means of eventrelated analyses of brain function during TP and TN items. $\mathrm{TP}$ and TN vs low-level fixation (X) contrasts ('loose comparisons') examine more general aspects of recognition memory performance, which are biased with respect to successful retrieval processes $(\mathrm{TP}>\mathrm{X})$ and encoding processes $(\mathrm{TN}>\mathrm{X})$, respectively (Buckner et al, 1998). In contrast TP $\langle>\mathrm{TN}$ contrasts ('tight comparisons') provide additional information concerning specific subcomponent processes during retrieval: $\mathrm{TP}>\mathrm{TN}$ contrasts specifically examine brain areas where signal intensity during successful retrieval of familiar information was significantly higher than signal intensity during successful rejection/encoding of new information. Areas of significant signal differences are, therefore, thought to represent 'successful retrieval' processes. Conversely, the reverse contrast TN $>\mathrm{TP}$ examines brain function related to 'encoding during attempted retrieval' (Daselaar et al, 2003). Contrast images were resampled to $2 \times 2 \times 2 \mathrm{~mm}$ in standard space and fed into in a second-level statistical analysis to examine effects of raloxifene treatment on (distinct aspects of) brain function during retrieval.

Activation images of main effects during recognition were produced by calculating an average activation map based on all individual activation maps for each session, medication regime, and event-type separately (ie BL (placebo), BL (raloxifene), TR (placebo), and TR (raloxifene)) in a mixed effects higher level analysis (Woolrich et al, 2004) using clusters determined by $Z<2.3$ and a corrected cluster significance threshold of $p=0.05$ (Forman et al, 1995; Friston et al, 1994; Worsley et al, 1992). Main effects at baseline were first tested for significant differences between raloxifene and placebo groups using the following contrasts: $\mathrm{BL}(\mathrm{RAL})\langle>\mathrm{BL}$ (PLAC), at $Z=3.1(p<0.001)$. Effects of treatment were then calculated using a repeated measures model, in which two explanatory variables (EVs) contrasted $\mathrm{BL}$ and TR sessions of placebo and raloxifene groups, allowing separate variances for both groups. Two additional EVs (one for each treatment group) coded for effects of test version on signal response. The main contrasts of interest were (TR (raloxifene)-BL (raloxifene)) \langle\rangle (TR (placebo)-BL (placebo)), testing for an interaction between raloxifene and placebo groups before and after treatment. Analyses were performed using a threshold of $Z=3.1 \quad(p<0.001)$. Images were rendered on a mean anatomical brain volume of all subjects in standard space (Talairach and Tournoux, 1988) for display purposes.

\section{RESULTS}

\section{Demographics}

Mean age of subjects was 64.1 (SD 2.4) years in the placebo group and 63.1 (SD 2.5) years in the raloxifene group. The placebo group contained two smokers $v s$ one in the raloxifene group. Education level was equal in both groups $\left(\chi^{2}=0.26 ; p=0.88\right)$.

\section{Subject Compliance and Discontinuation}

One subject (placebo group) was claustrophobic and data quality of both sessions was poor in another subject (raloxifene group). These data were discarded, yielding 28 complete data sets containing $\mathrm{BL}$ and TR data $(n=14$ raloxifene group, $n=14$ placebo group). Rescanning was performed once in a single individual (placebo group) because of poor data quality during the second scanning session. Subject compliance was good as assessed by tablet counts and there were no dropouts. No significant side effects were reported.

\section{Task Performance}

Numbers and percentages of all response types (ie TP, TN, $\mathrm{FP}$, and FN responses) are shown in Table 1A. Mean overall recognition accuracy was 0.60 (SD 0.20 ) above $50 \%$ chance level, with mean overall reaction time $1.63 \mathrm{~s}$ (SD $0.33 \mathrm{~s}$ ). Table 1B displays the significance of the difference between mean performance values (accuracy, latency and response bias) across both groups and scanning sessions. The placebo group showed significantly better mean recognition 
Table I Performance Measures in Raloxifene and Placebo Groups Before and After Treatment

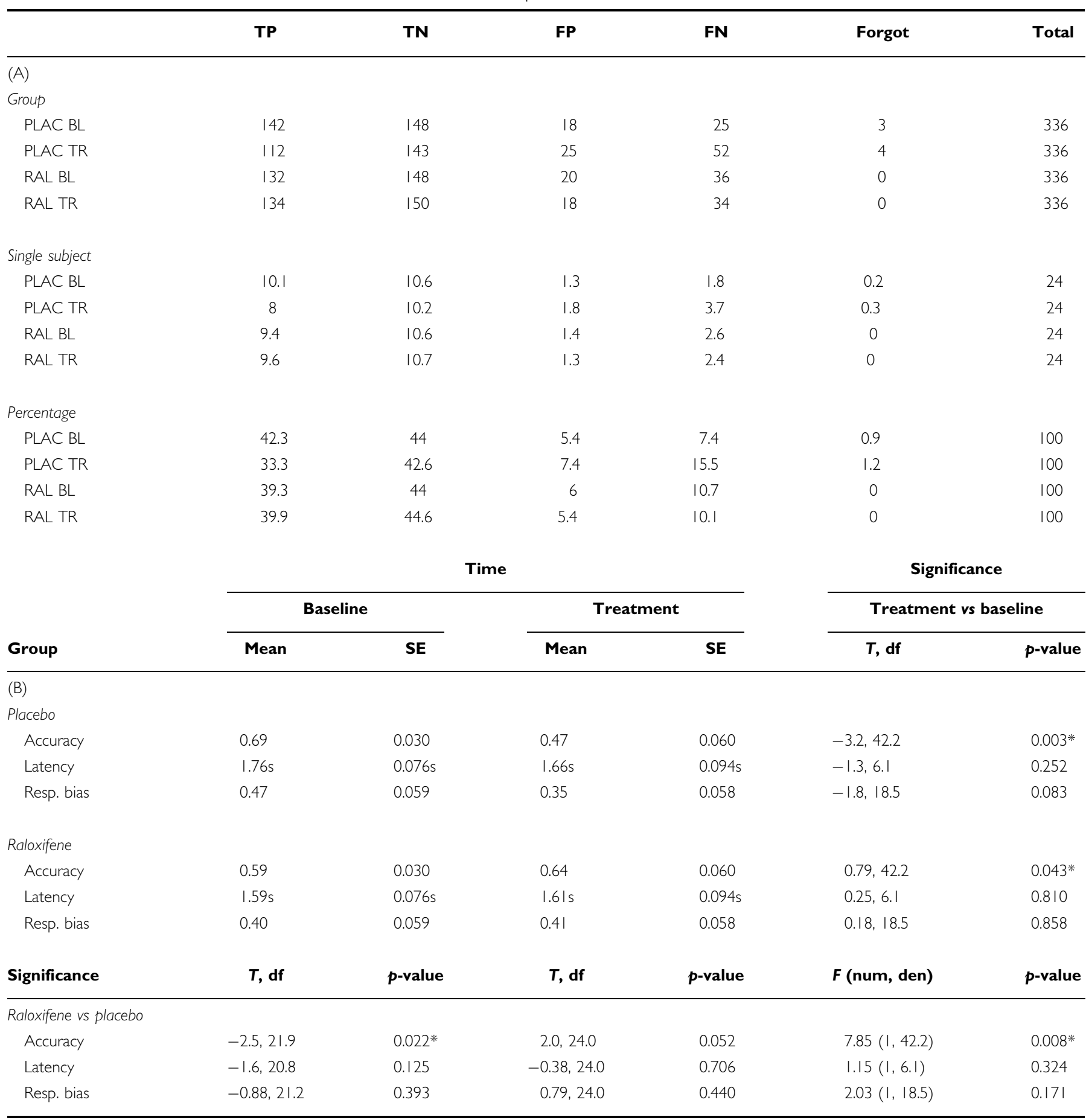

(A) Numbers of hits and misses during face recognition. 'Group', numbers for all subjects; 'Single subject', average numbers for single subjects; '\%', percentage of hits and misses for single subjects and group; 'PLAC', placebo; 'RAL', raloxifene; 'BL', baseline; 'TR', treatment (3 months); 'TP', correct recognitions (true positives); 'TN', correct rejections (true negatives); 'FP', false recognitions (false positives); 'FN', false rejections (false negatives); 'Forgot', total number of cases in which presentation of a face was not followed by a key-press; 'Total', total number of presented items. (B) Results of statistical analysis of task performance data (recognition accuracy, response latency, response bias) specified by group and time point of treatment. 'Group': treatment group (raloxifene, placebo). 'Time', time relative to onset of treatment (baseline, 3 months of treatment); 'Accuracy', recognition accuracy score (Pr); 'Latency', response latency (reaction time); 'Resp. Bias', response bias (Br); 'Mean', mean value of statistic; 'SE', standard error of statistic; 'Significance Raloxifene vs Placebo', significance of the effect of the factor 'group', testing for a difference between raloxifene and placebo groups; 'Significance treatment vs baseline': significance of the effect of the factor 'regime', testing for any effect of treatment; 'T, df: $T$-value, with corresponding degrees of freedom for the relevant comparison (contrast). ' $F$ (num, den)', $F$-value, with numerator and denominator for the relevant comparison; ' $p$-value', $p$-value for the relevant comparison. Bottom right boxes show results of statistical analysis of the effect of the interaction group*time, testing for a significant effect of raloxifene treatment vs palcebo intake. Asterisks (*) indicate effects significant at the 0.05 level. See text for further details. 
accuracy scores at baseline than the raloxifene group $(p=0.022)$. This trend was reversed after treatment $(p=0.052)$. After treatment, recognition accuracy increased significantly in the raloxifene group $(p=0.046)$, but decreased in the placebo group $(p=0.003)$, with the interaction being significant at $p=0.003$. Response latency was not significantly affected in both groups. Response bias showed a trend toward a decrease in the placebo group $(p=0.08)$, but remained stabile in the raloxifene group $(p=0.86)$, with the interaction being nonsignificant $(p=0.17)$. No significant effects were found for the covariate test version, or its interactions with other factors, on measures of task performance.

\section{Brain Function}

Main effects of face recognition (correct responses) involved activation of ventral and dorsal occipital (visual) areas, bilateral inferior parietal, parahippocampal, superior temporal, prefrontal areas, and the lateral sulci (Figure 1, Table 2). These effects were very similar to previous patterns of brain function observed during delayed recognition memory performance (Rugg et al, 2002). Activation patterns for TP and TN decisions differed significantly: TP $>$ TN contrasts showed activation bilaterally in primary visual cortex, anterior and posterior cingulate cortex, inferior parietal lobes, and insula, whereas unilateral activation was observed in right motor cortex, right basal ganglia, left cerebellum, and left inferior, middle, and superior frontal cortex $(Z>2.3$ cluster corrected (cluster threshold $Z=3.1)$; Figure 1). The lateralization in precentral areas likely represents increased motor activity of the right hand and fingers during TP decisions when compared to TN decisions. In contrast, only left motor cortex showed increased activation in $\mathrm{TN}>\mathrm{TP}$ contrasts (Figure 1).

The main contrast examining the interaction between raloxifene and placebo groups at baseline and after treatment showed significant increases in activation during TP decisions only. Effects occurred in the left parahippocampal area and right inferior prefrontal cortex (Figure 2c; Table $3 ; Z>3.1)$. Nearly identical effects were found on brain function during TN decisions, but these were not significant at $Z>3.1$ (Figure $2 \mathrm{~d} ; Z>2.3$ ). When studying the separate contributions of each group to treatment effects reported in Figure 2, the raloxifene group contributed mostly to the observed effects of treatment (Figure 2a and b). No significant effects were found of placebo intake on brain function. No significant treatment effects were found on contrasts describing TP $\langle>\mathrm{TN}$ activation differences. No decreases in activation were observed after treatment with either raloxifene or placebo. No significant differences were found at baseline between activation levels of raloxifene and placebo groups within areas of significant treatment effects $(Z>3.1)$. Plots of percent signal change (from global mean values) of peak voxels of local maxima illustrate intensity changes in both groups before and after treatment (Figure 2).

\section{DISCUSSION}

In a previous study, we reported effects of raloxifene treatment on brain activation during encoding of unfamiliar faces (Goekoop et al, 2005). Results of the current study complement our previous findings by examining brain activation during recognition of the encoded items.

\section{Effects of Raloxifene Treatment: Behavioral Data}

An unexpected drop in recognition accuracy was observed in the placebo group after 3 months of treatment, whereas performance in the raloxifene group increased slightly (Table 1). The nature of this drop in accuracy scores is unclear, but a number of explanations are possible. First, although group size of the current study is large to fMRI standards, it is rather small in terms of behavioral studies. Such small groups may show random differences in mean performance measures (Wilkinson and Halligan, 2004). Second, a drop in recognition accuracy at time 2 (retest phase) with respect to time 1 (test phase) is a regularly observed phenomenon in test-retest studies. Such effects are attributed to changes in context between test- and retest phases, and may involve 'true' decreases in sensitivity to old or new items as well as a change in response bias (ie subjects perform worse when they are insecure, or in an unfamiliar environment) (Feenan and Snodgrass, 1990). We therefore considered the possibility that a change in response bias was responsible for the observed decline in performance accuracy in the placebo group. A trend was observed for a difference in response bias $(\mathrm{Br})$ in the placebo group $(p=0.08)$, but no significant effects of raloxifene $v s$ placebo treatment were found. The possibility, therefore, exists that a change in response bias explains the observed decline in performance in the placebo group, but group size may not have been large enough to produce a significant difference. A change in response bias may reflect the subject's sensitivity to interference by changes in context between encoding and retrieval phases, practicing and scanning stages, or between first and second scanning sessions (eg no practicing round was held the day before the second scanning session). Since test items did not overlap between different test versions and the factor 'testversion' was no significant confounder of task performance scores, we consider it unlikely that specific test items or the use of different test versions acted as significant distracters. Since both groups were matched with respect to age, gender, and education level, we considered problems of group matching to be an unlikely source of performance differences between raloxifene and placebo groups. Additionally, a pathological decline of memory performance in the placebo group was considered unlikely, since these changes in performance occurred over a relatively short period of time ( 3 months) in otherwise healthy subjects (Goekoop et al, 2005). Future studies may want to include more subjects and perform more detailed assessment of cognitive status (including measures of context-dependency) in order to evaluate the effects of pharmacological substances on brain function and mental performance.

\section{Effects of Raloxifene Treatment: Functional-Behavioral Relationships}

Previous studies have shown that (small) behavioral changes do not necessarily translate into functional effects observed using fMRI at current group sizes, and vice versa 


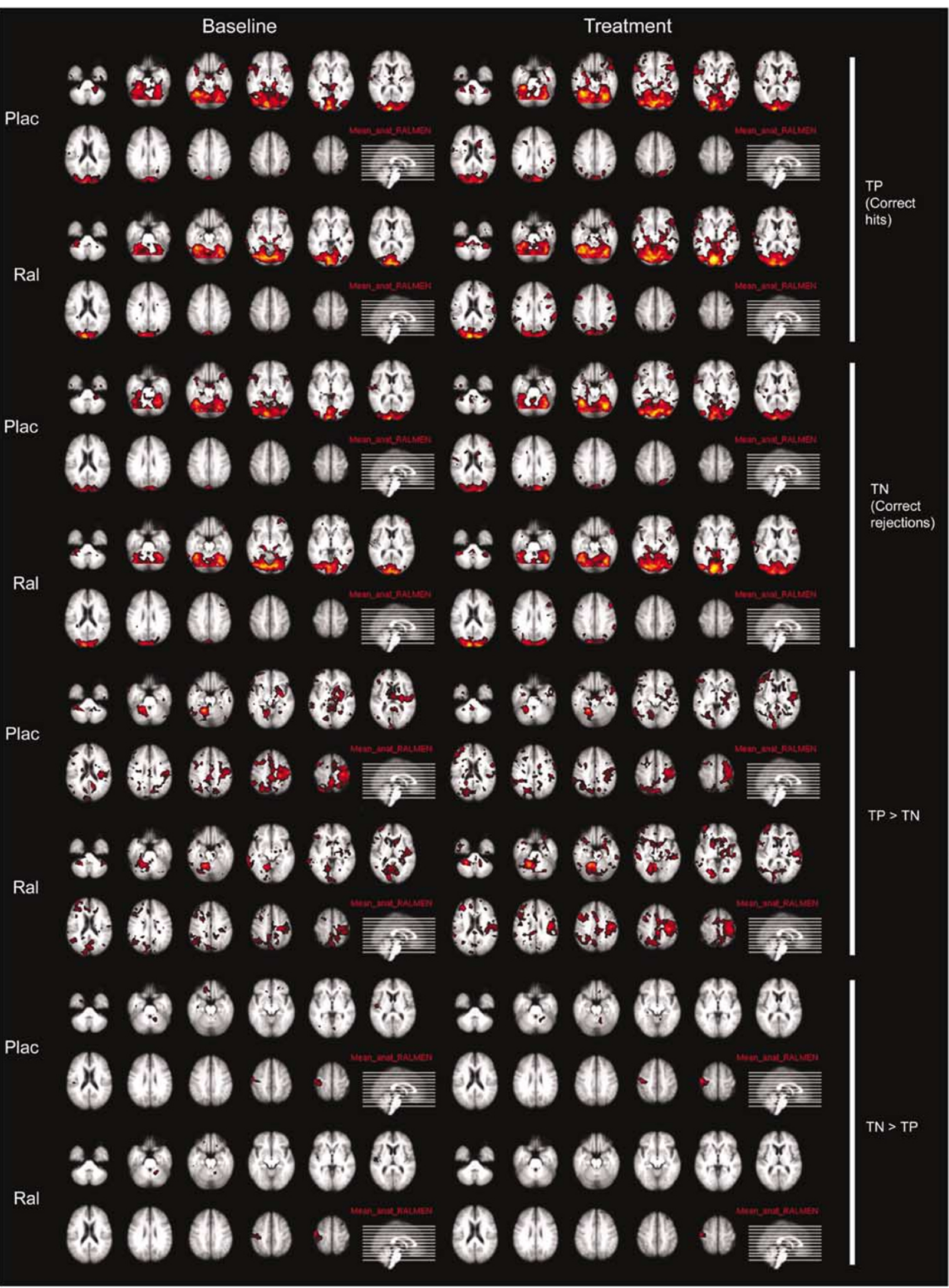

Figure I Axial slices showing main effects during face recognition rendered on a mean anatomical brain volume of all subjects. Left in the image is left in the brain. Effects after cluster correction at $Z=2.3$ and $p<0.05$. Color scale extends from $Z=2.3$ (red) to $Z=8.3$ (yellow). Effects during correct hits (TP), correct rejections (TN), and TP $\langle>$ TN activation differences. Baseline: brain activation at baseline. Treatment: brain activation after 3 months of treatment Ral: raloxifene group. Plac: placebo group. Visual inspection suggests an enhancement of activation after raloxifene treatment for TP items. See also text and Table 2. 
Table 2 Volume, Z-Scores, and Coordinates of Peak Voxels of Local Maxima of Main Effects during Correct Recognition of Familiar Items (TP Responses; Averaged Across Baseline and Treatment Sessions of Raloxifene and Placebo Groups)

\begin{tabular}{llrrrll}
\hline Nr. Vox & $\mathbf{Z}$ & $\boldsymbol{x}$ & $\boldsymbol{y}$ & $\mathbf{z}$ & Le/Ri & Region \\
\hline & 9.7 & 2 & -82 & 4 & $\mathrm{R}$ & Lingual gyrus \\
& 8.3 & -31 & -73 & -10 & $\mathrm{R}$ & Fusiform gyrus \\
8 & -30 & -72 & -12 & $\mathrm{~L}$ & Fusiform gyrus \\
& 7.1 & 25 & -35 & -2 & $\mathrm{R}$ & Parahippocampal gyrus \\
& 6.6 & -23 & -38 & 2 & $\mathrm{~L}$ & Parahippocampal gyrus \\
6 & 50 & 8 & -3 & $\mathrm{R}$ & Superior temporal gyrus \\
& 6 & 48 & 10 & -2 & $\mathrm{R}$ & Superior temporal gyrus \\
Confluent areas & 5.7 & -40 & 4 & 0 & $\mathrm{~L}$ & Lateral sulcus \\
& 5 & 0 & -40 & 60 & $\mathrm{R}$ & Paracentral lobe \\
4.7 & -38 & -62 & 40 & $\mathrm{R}$ & Inferior parietal lobe \\
4.2 & -35 & 10 & 39 & $\mathrm{~L}$ & Inferior parietal lobe \\
4.2 & 40 & 0 & 0 & $\mathrm{R}$ & Lateral sulcus \\
4 & 43 & 28 & 40 & $\mathrm{R}$ & Middle frontal gyrus \\
4 & -40 & 28 & 42 & $\mathrm{~L}$ & Middle frontal gyrus \\
3.9 & -39 & -10 & 54 & $\mathrm{~L}$ & Precentral gyrus \\
3.7 & -42 & -23 & 55 & $\mathrm{~L}$ & Postcentral gyrus
\end{tabular}

Similar regions were activated during TN responses. Effects after cluster correction at $Z=2.3$ and $p<0.05$. 'Nr. Vox', number of voxels in local maximum. No data are provided since all areas are confluent. ' $Z$ ', $Z$-score of peak voxel. ' $x, y, z$ ', coordinates of peak voxel in left-to-right, anterior-toposterior, and ventral-to-dorsal directions, respectively $(\mathrm{mm}$, Talairach convention); 'Le/Ri', left or right hemisphere. See also text and Figure I.

(Wilkinson and Halligan, 2004). To examine whether performance changes in each group separately were in any way related to the observed functional effects of treatment, we studied the separate contributions of raloxifene and placebo groups to the overall pattern of treatment effects on brain function during recognition. Treatment with placebo had no significant effect on brain activation. Indeed, treatment effects were mainly due to effects of raloxifene intake (Figure 2a and b). This indicated that the drop in performance observed in the placebo group was not a significant confounder of functional effects of raloxifene treatment $v s$ placebo as reported in this study (Figure 2c and d). Similarly, we examined the separate contributions of both groups to effects of raloxifene intake $v s$ placebo on brain activation during encoding in our previous study (Goekoop et al, 2005). Only the raloxifene group showed significant increases in brain activation in treatment-related areas, again indicating that placebo intake and subsequent performance changes were not a significant confounder of treatment effects during encoding.

Although further studies are necessary to corroborate our current findings, our data are consistent with the hypothesis that a 'normal' decrease in performance accuracy as a result of the subjects' sensitivity to a difference in context between test- and retest phases was countered by an active process related to raloxifene treatment (Goekoop et al, 2005). A reduction in the variability of recognition accuracy scores has been observed previously in elderly women receiving estrogen treatment (Wegesin and Stern, 2004). Additionally, a canceling of learning effects has been observed in male subjects receiving testosterone injections (Wolf et al, 2000), which may involve estrogen-mediated mechanisms (Wolf, 2003; Longcope et al, 1969). Such effects may involve effects of estrogens on arousal and attentional levels rather than direct effects on episodic memory. Estrogens are thought to influence brain function through a context-dependent effect on cortical arousal (Morgan et al, 2004). Such effects involve well-documented effects of estrogens on the four primary neuromodulatory neurotransmitters that regulate cortical arousal states (ie serotonin, dopamine, nor-adrenaline, and acetylcholine) (Bernardi et al, 2003; Korol, 2004). An increase in cholinergic (or noradrenergic) arousal is known to reflect an increase in signal-to-noise levels in neural networks, which translates into enhanced attention and working memory performance in animals and humans (Sarter et al, 2005). This mechanism of action is thought to be an important factor underlying improvement of memory performance in patients with $\mathrm{AD}$ that are treated with cholinesterase inhibitors (Sarter et al, 2005). Similarly, raloxifene treatment may have improved memory performance by enhancing cortical arousal (signal-to-noise-levels) during initial encoding, thereby reducing sensitivity to interference as a result of context-changes. Future studies may want to examine effects of raloxifene treatment on physiological measures of arousal and attention in order to examine this hypothesis in more detail.

\section{Effects of Raloxifene Treatment: Encoding Vs Recognition}

During face encoding, signal intensity during task performance was enhanced symmetrically across a wide range of neocortical areas (Goekoop et al, 2005). This rather generalized enhancement of cortical brain activation during face encoding was interpreted as a global effect of raloxifene intake on cortical arousal, rather than a specific effect of raloxifene treatment on all cognitive domains represented by this pattern (Goekoop et al, 2005). Treatment effects observed in the current study showed both similarities and differences with treatment effects during initial encoding. During both phases of memory performance, raloxifene intake produced increases in brain activation. Although decreases may have been possible in theory, these were not observed. The reason for the absence of signal decreases is unclear, but may reflect effects of increased arousal, since enhanced arousal levels increase rather than decrease the reactivity of neural networks (Coull, 1998; Morgan et al, 2004). In contrast to face encoding, however, treatment effects during face recognition did not involve a widespread increase of main effects of task performance in cortical structures. Direct effects of cortical arousal may, therefore, not have been relevant to treatment effects during face recognition. Indeed, a recent study shows that noradrenaline and neural steroids such as cortisol may modulate memory consolidation by interacting with arousal levels at initial encoding, rather than recognition (Cahill and Alkire, 2003). Although raloxifene intake may have influenced brain function during recognition independently of encoding, it is possible that the observed effects of raloxifene treatment during recognition represent indirect, or 'downstream' effects of treatment effects during initial encoding, 


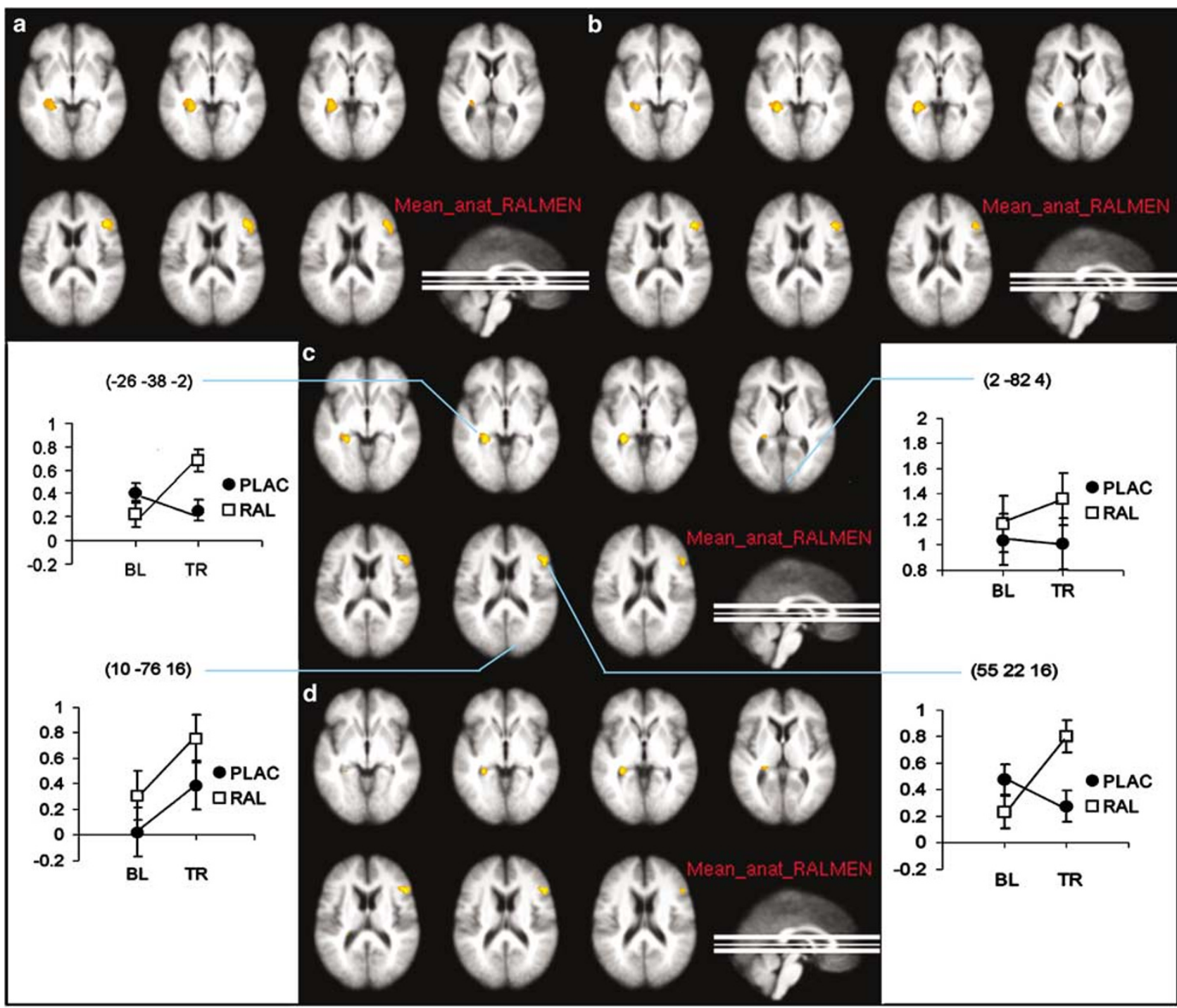

Figure 2 Axial slices showing effects of raloxifene treatment on brain function during TP and TN responses (correct hits and correct rejections). Functional maps are rendered on a mean anatomical brain volume of all subjects (Mean_anat_RALMEN). Left in the image is left in the brain. Color scale extends from $Z=2.3$ (orange) to $Z=4.0$ (yellow) for display purposes. No significant effects were observed after placebo intake (data not shown). (a) Raloxifene group, TP items, contrast TR $>$ BL. When compared to baseline, raloxifene treatment significantly increases brain activation in parahippocampal and right inferior prefrontal cortex. Effects are significant at $Z>3$.l. (b) Raloxifene group, TN items, contrast TR $>B L$ (RAL). Effects are not significant at $Z>3$. I, but are highly similar to treatment effects during TP responses at $Z>2.3$. (c) Raloxifene group vs placebo group, TP items, contrast (TR $>B L$ $(R A L))>(T R>B L(P L A C)$ ) (interaction). Increased activation is observed in parahippocampal cortex and right inferior prefrontal cortex (areas listed in Table 3). Effects are significant at $Z>3.1$. (d) Raloxifene group vs placebo group, TN items, contrast (TR $>B L(R A L))>(T R>B L(P L A C)$ ) (interaction). Effects are not significant at $Z>3$. I, but are highly similar to treatment effects during TP responses at $Z>2.3$. The strong resemblance of treatment effects on TP and TN contrasts suggests a general effect of raloxifene treatment on brain function during recognition, rather than a selective effect on encoding or retrieval processes (see text). Plots: graphs depicting the interaction between mean percent signal change of raloxifene and placebo groups at baseline and after treatment, as observed in peak voxels of local maxima of significant effects of treatment. Two reference voxels have been sampled in peak voxels of local maxima during face recognition in similar slices $(10-76$ I6) and $(2-824)$, showing no significant interaction after treatment. Means and standard deviations (error bars) are shown. Arrows indicate the corresponding clusters of activation. See also text and Table 3.

or consolidation processes occurring between the two tasks. Clearly, more research is needed to examine the neurodynamic changes underlying the observed effects of treatment, along with the possible interactions between treatment effects occurring at different stages of memory performance.

The current study shows that raloxifene treatment eventually affects brain function during memory retrieval. Since identical procedures of functional data analysis were followed, the asymmetric distribution of treatment effects across encoding and retrieval phases of memory perfor- mance suggests that the effects of raloxifene, like the effects of many other pharmacological substances, are processspecific (Honey and Bullmore, 2004). A direct statistical comparison of treatment effects between encoding and recognition phases was not performed, however, since these tasks were too dissimilar (ie block $v s$ event-related designs) to allow meaningful comparisons. We therefore report our findings of the effects of cholinergic challenge separately for both memory tasks. Although encoding processes during attempted retrieval may differ from encoding during 
Table 3 Volume, Z-Scores, and Coordinates of Peak Voxels of Local Maxima of Significant Effects of Medication Intake $(Z>3.1)$ As Determined by the Interaction Between Activation Levels of Raloxifene and Placebo Groups at Baseline and After Treatment (see Methods)

\begin{tabular}{lcccccl}
\hline Nr. Vox & $\boldsymbol{Z}$ & $\boldsymbol{x}$ & $\mathbf{y}$ & $\mathbf{z}$ & Le/Ri & Region \\
\hline 33 & 3.53 & -26 & -38 & -2 & $\mathrm{~L}$ & Parahippocampal area \\
16 & 3.51 & 52 & 22 & 16 & $\mathrm{R}$ & Inferior frontal gyrus \\
\hline
\end{tabular}

'Nr. Vox', number of voxels in local maximum (voxel size $2 \times 2 \times 2 \mathrm{~mm}$ ); ' $Z$ ', $Z$ score of peak voxel; ' $x, y, z$ ', coordinates of peak voxel in left-to-right, rostral-tocaudal, and ventral-to-dorsal directions, respectively ( $\mathrm{mm}$, Talairach convention); 'Le/Ri', left or right hemisphere. See also text and Figure 2.

attempted encoding (Reber et al, 2002; Rombouts et al, 2001), studies of brain function during retrieval allow analyses of encoding and retrieval processes within the same scanning session (see Methods), which avoids some of the potential confounds that may be introduced by acrosssession comparisons (eg differences in task design, subject positioning, motion artefacts).

Significant effects of raloxifene treatment were found on brain activation during TP decisions (familiar items), but not on TN decisions (unfamiliar items) (Figure 2c). Although this may indicate a preference for raloxifene treatment to influence successful recognition rather than encoding during attempted retrieval, the small number of items analyzed per subject somewhat limit these findings (Table 1). Indeed, when the threshold for significant brain function was lowered for contrast images representing treatment effects during TN decisions, treatment effects were found that were nearly identical to those observed for TP decisions (Figure 2d). This suggested that raloxifene treatment affected more general aspects of recognition memory function that are examined equally by 'loose' comparisons of brain function during TP and TN decisions $v s$ fixation (see Methods). In order to explore this possibility in more detail, we studied the effects of raloxifene on 'tight' $\mathrm{TP}\langle\rangle \mathrm{TN}$ comparisons of brain function, which examine specific encoding and retrieval processes during task performance (see Methods). No significant effects of raloxifene treatment were found, supporting the view that raloxifene treatment affected general aspects of recognition memory performance, rather than specific subcomponent processes during retrieval. Brain areas showing treatment effects for TP items included the right inferior frontal gyrus and left parahippocampal cortex (Figure 2c, Table 3). These structures are important to retrieval of episodic (visuospatial) information after some delay (Schacter and Wagner, 1999; Fletcher and Henson, 2001; Rugg et al, 2002). Enhanced activation of these structures may reflect direct effects of estrogen receptor stimulation on neural signaling and brain function (Bisagno et al, 2003), or sustained effects of estrogen receptor stimulation, which alters protein synthesis and enhances the outgrowth of neural spines in hippocampal areas, which is known to be associated with increased memory performance (Li et al, 2004). Future studies may want to examine the relationships between functional effects of treatment and changes in (parahippocampal) spine density in vivo, by combining phMRI and molecular imaging techniques (eg PET). Based on the current results, we predict an effect of raloxifene treatment on delayed (visuospatial) memory performance in males.

\section{Effects of Raloxifene Treatment: Blood Vs Brain}

In the absence of direct measurements of vascular changes (eg perfusion studies), only indirect arguments may serve to locate treatment effects on blood oxygenation level-dependent (fMRI) signal reactivity more precisely to either the neural or vascular compartment (Honey and Bullmore, 2004). In the current study, vascular effects seemed less likely than neurogenic effects for several reasons. First, effects on blood vessels are likely to be similar across different mental processes, yet treatment effects differed between encoding and retrieval stages of memory performance. Second, effects on vascular tissue are likely to be generalized instead of localized, yet treatment effects were localized in our studies. Even during encoding, which showed a widespread pattern of signal enhancement, there were some highly vascularized and active brain structures (eg primary visual cortex) that did not show enhancement of signal intensity after raloxifene treatment. Finally, treatment effects were found in functionally meaningful areas only (eg not in white matter). Together, these observations strongly suggest that the effects of raloxifene treatment were of neurogenic rather than of vascular origin.

\section{Summary and Conclusions}

Raloxifene treatment enhanced brain activation in male subjects during recognition of familiar items. A possible treatment mechanism involves enhanced memory retrieval as a result of increased cortical arousal during initial encoding, which reduces effects of context-dependency. Similar effects may underlie the ability of long-term raloxifene treatment to delay the onset of MCI in elderly women. Our combined studies on the effects of raloxifene treatment on episodic memory performance in male subjects predict the occurrence of small behavioral effects on working memory and delayed (visuospatial) memory performance, executive functions and verbal skills, which are secondary to enhanced arousal and attention during initial encoding. Further neuropsychological studies involving larger groups of subjects are necessary to test the validity of these predictions.

\section{ACKNOWLEDGEMENTS}

We thank Stichting Alzheimer and the Neuropsychiatrie Foundation for financial support, and Ans Nicolaas-Merkus for subject recruitment. Serge Rombouts is supported by a grant from the Netherlands Organization for Scientific Research (NWO; Grant No. 916.36.117).

\section{REFERENCES}

American Psychiatric Association (2000). Diagnostic and Statistical Manual of Mental Disorders DSM-IV-TR (Text Revision), 4th edn. American Psychiatric Association: Washington, DC. ISBN 0890420254.

Barrett-Connor E, Grady D, Sashegyi A, Anderson PW, Cox DA, Hoszowski K et al (2002). Raloxifene and cardiovascular events in osteoporotic postmenopausal women - four-year results from 
the MORE (Multiple Outcomes of Raloxifene Evaluation) randomized trial. JAMA 287: 847-857.

Bernardi F, Pluchino N, Stomati M, Pieri M, Genazzani AR (2003). CNS: sex steroids and SERMs. Ann NY Acad Sci 997: 378-388.

Bisagno V, Bowman RE, Luine VN (2003). Functional aspects of estrogen neuroprotection. Endocrine 21: 33-41.

Blum A, Hathaway L, Mincemoyer R, Schenke WH, Csako G, Waclawiw MA et al (2000). Hormonal, lipoprotein, and vascular effects of the selective estrogen receptor modulator raloxifene in hypercholesterolemic men. Am J Cardiol 85: 1491-1494.

Buckner RL, Koutstaal W, Schacter DL, Dale AM, Rotte M, Rosen BR (1998). Functional-anatomic study of episodic retrieval II - selective averaging of event-related fMRI trial to test the retrieval success hypothesis. Neuroimage 7: 163-175.

Buckner RL, Wheeler ME, Sheridan MA (2001). Encoding processes during retrieval tasks. J Cogn Neurosci 13: 406-415.

Cahill L (2003). Sex-related influences on the neurobiology of emotionally influenced memory. Amygdala Brain Funct: Basic Clin Approach 985: 163-173.

Cahill L, Alkire MT (2003). Epinephrine enhancement of human memory consolidation: interaction with arousal at encoding. Neurobiol Learn Mem 79: 194-198.

Corwin J (1994). On measuring discrimination and response bias: unequal numbers of targets and distractors and two classes of distractors. Neuropsychology 8: 110-117.

Coull JT (1998). Neural correlates of attention and arousal: insights from electrophysiology, functional neuroimaging and psychopharmacology. Prog Neurobiol 55: 343-361.

Daselaar SM, Veltman DJ, Rombouts SARB, Raaijmakers JGW, Jonker C (2003). Neuroanatomical correlates of episodic encoding and retrieval in young and elderly subjects. Brain 126: 43-56.

Doran PM, Riggs BL, Atkinson EJ, Khosla S (2001). Effects of raloxifene, a selective estrogen receptor modulator, on bone turnover markers and serum sex steroid and lipid levels in elderly men. J Bone Miner Res 16: 2118-2125.

Feenan K, Snodgrass JG (1990). The effect of context on discrimination and bias in recognition memory for pictures and words. Mem Cognit 18: 515-527.

Fletcher PC, Henson RNA (2001). Frontal lobes and human memory-insights from functional neuroimaging. Brain 124: 849-881.

Forman SD, Cohen JD, Fitzgerald M, Eddy WF, Mintun MA, Noll DC (1995). Improved assessment of significant activation in functional magnetic-resonance-imaging (Fmri) - use of a clustersize threshold. Magn Reson Med 33: 636-647.

Friston KJ, Worsley KJ, Frackowiak RSJ, Mazziotta JC, Evans A (1994). Assessing the significance of focal activations using their spatial extent. Hum Brain Mapp 1: 210-220.

Goekoop R, Duschek EJJ, Knol DL, Barkhof F, Netelenbos C, Scheltens $\mathrm{P}$ et al (2005). Raloxifene exposure enhances brain activation during memory performance in healthy elderly males; its possible relevance to behavior. Neuroimage 25: 63-75.

Heringa M (2003). Review on raloxifene: profile of a selective estrogen receptor modulator. Int J Clin Pharmacol Therap 41: 331-345.

Honey G, Bullmore E (2004). Human pharmacological MRI. Trends Pharmacol Sci 25: 366-374.

Jenkinson M, Bannister P, Brady M, Smith S (2002). Improved optimization for the robust and accurate linear registration and motion correction of brain images. Neuroimage 17: 825-841.

Jenkinson M, Smith S (2001). A global optimisation method for robust affine registration of brain images. Med Image Anal 5: $143-156$

Korol DL (2004). Role of estrogen in balancing contributions from multiple memory systems. Neurobiol Learn Mem 82: 309-323.

Li CJ, Brake WG, Romeo RD, Dunlop JC, Gordon M, Buzescu R et al (2004). Estrogen alters hippocampal dendritic spine shape and enhances synaptic protein immunoreactivity and spatial memory in female mice. Proc Acad Natl Sci USA 101: 2185-2190.

Longcope C, Kato T, Horton R (1969). Conversion of blood androgens to estrogens in normal adult men and women. J Clin Invest 48: 2191-2201.

Maki PM, Resnick SM (2001). Effects of estrogen on patterns of brain activity at rest and during cognitive activity: a review of neuroimaging studies. Neuroimage 14: 789-801.

Morgan MA, Schulkin J, Pfaff DW (2004). Estrogens and nonreproductive behaviors related to activity and fear. Neurosc Biobehav Rev 28: 55-63.

Reber PJ, Siwiec RM, Gitleman DR, Parrish TB, Mesulam MM, Paller KA (2002). Neural correlates of successful encoding identified using functional magnetic resonance imaging. $J$ Neurosci 22: 9541-9548.

Rombouts SARB, Barkhof F, Witter MP, Machielsen WCM, Scheltens P (2001). Anterior medial temporal lobe activation during attempted retrieval of encoded visuospatial scenes: an event-related fMRI study. Neuroimage 14: 67-76.

Rugg MD, Otten LJ, Henson RNA (2002). The neural basis of episodic memory: evidence from functional neuroimaging. Phil Trans R Soc London B 357: 1097-1110.

Sarter M, Hasselmo ME, Bruno JP, Givens B (2005). Unraveling the attentional functions of cortical cholinergic inputs: interactions between signal-driven and cognitive modulation of signal detection. Brain Res Rev 48: 98-111.

Schacter DL, Wagner AD (1999). Medial temporal lobe activations in FMRI and PET studies of episodic encoding and retrieval. Hippocampus 9: 7-24.

Smith SM (2002). Fast robust automated brain extraction. Hum Brain Mapp 17: 143-155.

Smith SM, Jenkinson M, Woolrich MW, Beckmann CF, Behrens TEJ, Johansen-Berg $\mathrm{H}$ et al (2004). Advances in functional and structural MR image analysis and implementation as FSL. Neuroimage 23: S208-S219.

Talairach J, Tournoux P (1988). Co-Planar Stereotaxic Atlas of the Human Brain. Thieme Verlag: Stuttgart, Germany. ISBN 3137117011.

Thiel CM (2003). Cholinergic modulation of learning and memory in the human brain as detected with functional neuroimaging. Neurobiol Learn Mem 80: 234-244.

Wegesin DJ, Stern Y (2004). Inter- and intraindividual variability in recognition memory: effects of aging and estrogen use. Neuropsychology 18: 646-657.

Wilkinson D, Halligan P (2004). Opinion-the relevance of behavioural measures for functional-imaging studies of cognition. Nat Rev Neurosc 5: 67-73.

Wolf OT (2003). Cognitive functions and sex steroids. Ann D Endocrinol 64: 158-161.

Wolf OT, Preut R, Hellhammer DH, Kudielka BM, Schurmeyer TH, Kirschbaum C (2000). Testosterone and cognition in elderly men: a single testosterone injection blocks the practice effect in verbal fluency, but has no effect on spatial or verbal memory. Biol Psychiatry 47: 650-654.

Woolrich MW, Behrens TEJ, Beckmann CF, Jenkinson M, Smith SM (2004). Multilevel linear modelling for FMRI group analysis using Bayesian inference. Neuroimage 21: 1732-1747.

Woolrich MW, Ripley BD, Brady M, Smith SM (2001). Temporal autocorrelation in univariate linear modeling of FMRI data. Neuroimage 14: 1370-1386.

Worsley KJ, Evans AC, Marrett S, Neelin P (1992). A 3-dimensional statistical-analysis for Cbf activation studies in human brain. J Cereb Blood Flow Metabol 12: 900-918.

Yaffe K, Krueger K, Cummings SR, Blackwell T, Henderson VW, Sarkar S et al (2005). Effect of raloxifene on prevention of dementia and cognitive impairment in older women: the multiple outcomes of raloxifene evaluation (MORE) randomized trial. Am J Psychiatry 162: 683-690. 